\title{
Biblioteca digital: bibliografia internacional anotada
}

\section{Murilo Bastos da Cunha}

\section{Resumo}

Bibliografia internacional seletiva e anotada sobre bibliotecas digitais. Aborda os seguintes aspectos: a) visionários, principais autores que escreveram sobre a biblioteca do futuro, no período de 19451985; b) conceituação de biblioteca digital; c) projetos em andamento na Alemanha, Austrália, Brasil, Canadá, Dinamarca, Espanha, Estados Unidos, França, Holanda, Japão, Nova Zelândia, Reino Unido, Suécia e Vaticano; d) aspectos técnicos relativos à construção de uma biblioteca digital: arquitetura do sistema, conversão de dados e escaneamento, marcação de textos, desenvolvimento de coleções, catalogação, classificação/indexação, metadados, referência, recuperação da informação, direitos autorais e preservação da informação digital; e) principais fontes de informação: monografia, periódicos, reuniões técnicas específicas, lista de discussão, grupos e centros de estudos, cursos e treinamento.

Termos de indexação

Biblioteca digital; Arquitetura de biblioteca digital; Conversão de dados; Escaneamento, marcação de textos; SGML;

Desenvolvimento de coleções; Catalogação; Classificação; Documento digital;

Metadados; Recuperação da informação; Z39.50; Direitos autorais; Preservação da informação.

\section{INTRODUÇÃO}

Este trabalho tem por objetivo apresentar uma bibliografia anotada das principais fontes de informação relacionadas com bibliotecas digitais. Tendo em vista o enorme crescimento da literatura sobre o assunto, ocorrido nos últimos anos, esta bibliografia procurou ser seletiva, arrolando os documentos básicos e dados sobre os principais projetos de bibliotecas digitais em andamento nos mais diversos países. Procurou-se incluir, além dos dados referenciais tradicionais, o endereço na Internet (URL) dos documentos e instituições. Na busca dos dados foram consultados o Library and Information Science Abstracts (Lisa), o Information Science Abstracts (ISA), o "Library Literature" (LL), dezenas de páginas iniciais (home pages) e os acervos das bibliotecas da University of Michigan (US). Foram incluídos documentos em diversas línguas, havendo, entretanto, predominância da língua inglesa.

\section{BIBLIOGRAFIA}

Esta bibliografia está arranjada por grandes assuntos. Dentro de cada assunto segue-se a ordem alfabética dos autores e/ou instituições.

\section{A) VISIONÁRIOS}

Abaixo estão incluídos os principais autores que no período de 1945-1985 imaginaram a necessidade de haver mudanças na biblioteca tradicional e a criação de uma biblioteca que utilizasse recursos tecnológicos para fazer frente à explosão bibliográfica melhorando, por conseguinte, o acesso à informação por parte do usuário, principalmente aqueles das áreas científicas e tecnológicas.
Bush, Vannevar. As we may think. The Atlantic Monthly, n. 176, p. 101-108, July 1945.

URL: http://www.notredame.ac.jp/ftplib/ Articles/CMC/bush45.txt

URL: http://www.isg.sfu.ca/ duchier/ misc/bush

Neste documento, Bush imaginou uma máquina - denominada Memex - que facilitaria a disseminação da informação científica e, com o emprego dela, armazenaria, para uso posterior, toda a informação do seu interesse. Este autor é considerado o pai da biblioteca digital.

Dowlin, Kenneth E. The electronic library: the promise and the process. New York: Neal-Schuman, 1984.199 p. ISBN 091-8212-758

Apresenta a idéia da criação de uma biblioteca eletrônica dentro da Pikes Peak Library (Colorado), na qual seria enfatizado o rápido acesso à informação por meio de sistemas de comunicação eletrônica. A biblioteca pública se transformaria em um centro de informação comunitário, coletando informações sob as mais diversas formas, criando bases de dados relativas a assuntos sociais ligados à sua clientela.

Hill, Albert G. "The storage, processing and the communication of information". In: Bibliography in an age of Science. Urbana (IL): University of Illinois Press, 1951. p. 73-90.

Propõe a idéia de uma biblioteca eletrônica para facilitar o acesso à informação por parte do pesquisador que, mediante perfis de interesses armazenados no computador, poderia receber $e$ enviar informações relevantes para as suas atividades. 
Kemeny, John. A library for 2000 A. D. In: Greenberger, Martin. Computers and the world of the future. Cambridge (Mass.): MIT Press, 1965.

Afirma que a biblioteca universitária será obsoleta em torno do ano 2000 e que a biblioteca do futuro dependerá fortemente da automação e que o acesso à informação será feito por meio de redes automatizadas. Propõe, ainda, a criação de uma national research library que hospedaria os acervos das áreas de ciência e tecnologia.

Lancaster, F. W. Toward paperless information systems. New York: Academic Press, 1978. 179 p. ISBN 012-4360-505 Lancaster, F. W. Libraries and librarians in the age of electronics. Arlington (VA): Information Resources, 1982. 229 p. ISBN 087-8150-404

Nessas duas obras o autor comenta suas idéias sobre a sociedade sem papel (paperless society) na qual a biblioteca tradicional irá desaparecer, sendo substituída pela biblioteca digital.

Licklider, J. C. R. Libraries of the future. Cambridge (Mass.): MIT Press, 1965. $219 p$.

Seguidor das idéias de Vannevar Bush, sugeriu a criação de uma biblioteca eletrônica, tendo em vista que o livro era um suporte de informação inadequado.

Nelson, Theodor Holm.

URL: http://www.xanadu.net/xanadu/

Idealizador do hipertexto (em 1965), autor prolífico, propôs a criação de uma biblioteca eletrônica (World Publishing Repository - Project Xanadu), onde seriam armazenados os textos integrais de documentos. Xanadu seria um "lugar mágico da memória literária onde nada seria esquecido".

Parker, Edwin B. The new communication media. In: Wallia, C. S., ed. Toward century 21: technology, society and human values. New York: Basic Books, 1970.

Previu que até 1975 os catálogos em linha estariam disponíveis e que em 1985 todo o acervo bibliográfico estaria armazenado em computadores. Imaginou uma máquina que teria tela e teclado, contendo as funções de uma biblio- teca e de um jornal, com a capacidade de acessar redes de informação por meio da linha telefônica.

Taylor, Robert S. The making of a library; the academic library in transition. New York: Becker and Hayes, 1972. 250 p. ISBN 047-1848-31X

Propõe a necessidade de se abandonar o conceito tradicional de biblioteca como depositária da informação e adotar um novo conceito de comunicação científica que transcenda as estruturas físicas da biblioteca. Assim, a biblioteca universitária deveria deixar de ser um lugar físico para ser um processo que permearia todo o campus.

\section{B) CONCEITUAÇÃO}

A biblioteca digital é também conhecida como biblioteca eletrônica (principalmente no Reino Unido), biblioteca virtual (quando utiliza recursos da realidade virtual), biblioteca sem paredes e biblioteca cibernética.

Atkinson, R. Library functions, scholarly communication and the foundation of the digital libraries: laying claim to the control zone. Library Quartely, v. 66, p. 239-265, July 1996.

Discussão sobre as funções da biblioteca, a comunicação científica e os papéis a serem executados pela biblioteca digital.

Baldacci, Maria Bruna. La biblioteca del 2000: dal punto di vista delle biblioteche. Bolletino AIB, v. 33 , n. 4, p. 423436, Dicembre 1993.

As enormes redes interligando a maioria dos países tornaram factível a criação de uma biblioteca global. A autora aponta uma série de problemas que deverão ser solucionados para que se tenha uma verdadeira biblioteca digital.

Bauwens, Michel. De virtuele biblioteek. Cahiers de la Documentation, v. 46, n. 4, p. 175-183, 1992.

Explica o funcionamento de uma biblioteca virtual.
Billington, J.H. A technological flood requires human navigators. American Libraries, v. 27, n. 6, p. 39-40, 1996.

Comenta a contribuição da biblioteca pública na democratização da sociedade e o papel da biblioteca na sociedade da informação. Acredita que o novo bibliotecário deva ser um navegador do conhecimento (knowledge navigator).

Broering, N.C. Changing focus: tomorrow's virtual library. Serials Librarian, v. 25, n. 3/4, p. 73-94, 1995.

Discute o planejamento estratégico e o papel do bibliotecário em um ambiente acadêmico digital.

Cloyes, Kay. The journey from vision to reality of a virtual library. Special Libraries, v. 85, p. 253-255, Fall 1994.

Descrição dos serviços que serão prestados pela biblioteca digital. Drabenstott, Karen M. \& Burman, Celeste M. Analytical review of the library of the future. Washington (DC): Council on Library Resources, 1994. 200 p.

Revisão da literatura em língua inglesa sobre a evolução do conceito da biblioteca do futuro.

England, M., \& Shaffer, M. (1994). Librarians in the digital library. Annual Conference on the Theory and Practice of Digital Libraries, 1., 1994, College Station, Texas, 1994. Proceedings. College Station (TX): Texas A \& M University, Deparment of Computer Science, 1994. p. 211-212

Comenta as possíveis mudanças que ocorrerão com o bibliotecário no contexto da biblioteca digital, enfatizando novos papéis ligados ao ensino, consultoria, pesquisa e acesso à informação.

Graham, P. S. Requirements for the digital research library. College \& Research Libraries, v. 56, p. 331-339, July 1995. URL: http://aultnis.rutgers.edu/texts/ DRC.html

Autor mostra que, para atender às necessidades dos usuários, os fundadores da biblioteca digital devem cumprir duas tarefas: estabelecimento de um repositório de material eletrônico para a pesquisa e implementação de mecanismos para a sua plena utilização. 
Heinisch, Christian. CyberLib, vision and economic analysis. Libri, v. 44, n. 4, p. 393-399, 1994.

Descrição de uma biblioteca digital com ênfase no seus aspectos econômicos.

Kahin, B. Institutional and policy issues in the development of the digital library. 1994. (on-line).

URL: http://www.press.umich.edu/jep/ works/kahin.dl.html

Discute alguns problemas da biblioteca digital, a saber, compartilhamento de recursos e o controle da informação por meio dos direitos autorais.

Kochtanek, T. R. On the role of libraries in a virtual landscape. In: National Online Meeting, 16., 1995, New York. Proceedings. New York: Learned Information, 1995. p. 223-231.

Examina os termos biblioteca virtual e biblioteca digital e discute suas definições, compararando-os com a biblioteca tradicional. Descreve a evolução da biblioteca tradicional para a biblioteca digital e o papel do bibliotecário nesse ambiente futuro.

LaRue, J. The library tomorrow: a virtual certainty. Computers in Libraries, v. 13, n. 2, p. 14-17, 1993.

O autor sugere ao leitor reconsiderar o termo biblioteca virtual em relação ao ambiente da biblioteca pública na era digital. Conclui que a biblioteca pública, como unidade física, provê muitos serviços essenciais que não poderão ser fornecidos pela biblioteca virtual.

LeCodiac, Yves F. La mesure dans le centre de documentation électronique. Documentaliste Science de I'Information, v. 31, n. 2, p. 78-82, 1994.

Conceituação de uma biblioteca digital, denominada pelo autor de centro eletrônico de documentação.

Levy, D.M., Marshall, C. C. Going digital: A look at assumptions underlying digital libraries. Communications of the ACM, v. 38, n. 4, p. 77-84, 1995.

O autor argumenta que a atual definição de biblioteca digital é muito restrita e não irá satisfazer as necessidade fu- turas dos usuários. Assim, o mesmo propõe que haja uma integração das mídias (documentos híbridos), instrumentos de gerenciamento, desenvolvimento de novas técnicas de catalogação e métodos de manutenção de coleções.

Lyman, P. What is a digital library? Technology, intellectual property and the public interest. Daedalus, Jornal of the American Academy of Arts and Science, v. 125, n. 4, Fall 1996, p. 1-33.

Aborda os novos papéis da publicação eletrônica e da biblioteca no espaço cibernético.

Maignien, Y. La bibliotheque virtuelle ou de l'ars memoria à Xanadu. Bulletin Bibliothèques de France, v. 40, n. 2, p. 8-17, 1995.

Interessante análise da evolução da história da biblioteca, desde a época medieval até a biblioteca digital.

Miksa, F., \& Doty, P. Intellectual realities and the digital library. In: Annual Conference on the Theory and Practice of Digital Libraries, 1., 1994, College Station, Texas. Proceedings. College Station (TX): Texas A \& M University, 1994. p. 1-5.

URL: http://www.csdl.tamu.edu/DL94/ paper/miksa.html

Documento examina a definição de biblioteca e o conceito de biblioteca digital. As noções de coleção e fontes de informação são discutidas visando a questionar o conceito de biblioteca digital.

Newby, G. Digital library models and prospects. American Society for Information Science, Mid-year Meeting, San Diego (CA), May 18-22, 1996. Proceedings. Medford: Information Today, 1996. p. 101-111.

Aborda os aspectos econômicos da biblioteca digital e suas implicações junto ao mercado editorial.

Wiederhold, G. Digital libraries, value, and productivity. Communications of the ACM, v. 38, n. 4, p. 85-96, 1995.

O autor discute os novos meios de publicação, os serviços da biblioteca tradicional que irão desaparecer e os no- vos que surgirão. Identifica o gerenciamento do direito autoral, busca de imagem, livros dinâmicos e a garantia do acesso como novos serviços que serão providos pelas futuras bibliotecas.

Winograd, T. Conceptual models for comparison of digital library systems and approaches. Working Paper, 1995. URL: http://www-diglib.stanford.edu/ diglib/WP/PUBLIC/DOC13.html

Discute os modelos conceituais de biblioteca digital. Mostra as diferenças entre o modelo de dados (data model) e o modelo de atividades (activity model), bem como suas aplicações e problemas operacionais sob o ponto de vista da informática.

\section{C) PROJETOS EM ANDAMENTO}

Abaixo constam referências sobre os principais projetos de bibliotecas digitais ora em desenvolvimento, arranjados segundo a ordem alfabética por países. Dentro de cada país segue-se a ordem alfabética da instituição maior responsável pelo projeto.

\section{Alemanha \\ C 1.1 - German National Bibliography (GNB)}

URL: http://www.forwiss.tu-muenchen. de/ oewal

Dorr, M.; Haddouti, H. \& Wiesener, S. The German National Bibliography 16011700: digital images in a cooperative cataloging project. IEEE International Forum on Research and Technology Advances in Digital Libraries, ADL'97, May 7-9 1997, Washington, D. C. Proceedings. Washington (DC): IEEE Computer Society, 1997. p. 50-55.

Descreve o projeto de digitalização de imagens e do texto integral de obras raras da bibliografia alemã publicadas no século XVIII. 


\section{C1.2-OMNIS}

URL: http://www.forwiss.tu-muenchen. de/ vd17

Projeto pioneiro de biblioteca digital na Alemanha, desenvolvido em 1993 no FORWISS (Bavaria Research Center for Knowledge-Based Systems). Este sistema é utilizado por diversas bibliotecas alemãs.

Bayer, R.; Vogel, P.; Wiesener, S. OMNIS/Myriad document retrieval and its database requirements. In: International Conference on Database and Expert Systems Aplications (DEXA'94), $5^{\text {th }}$., Athens (Greece), September 5-9, 1994. Proceedings. Berlin; Springer-Verlag, 1994.

Descrição das características técnicas do sistema OMNIS.

\section{C1.3 - Project MeDoc}

URL: http://medoc.informatik. tumuenchen.de

Projeto, iniciado em setembro de 1995 , mediante esforço cooperativo entre a Sociedade Alemã de Informática (GI), FIZ Karlsruhe e Springer-Verlag, tem por objetivo fornecer acesso a texto completo da literatura de informática.

\section{C2 - Austrália}

Ianella, Renato. Australian Digital Libraries Initiatives. D-Lib Magazine, December 1996.

URL: www.dlib.org/dlib/december96/ 12ianella.html

Descrição dos projetos de bibliotecas digitais ora em andamento na Austrália.

\section{C2.1 - Australian Cooperative Digiti- sation Project}

URL: http://www.nla.gob.au/ferg/ fergproj.html

Coleman, R. Australian co-operative digitisation project, 1840-45. Ariadne n. 8, 1997.

URL: http://www.ariadne.ac.uk/issue8/ digitisation/
Descreve o Australian Cooperative Digitisation Project que tem por objetivo a digitalização de periódicos e obras de ficção de interesse para a Austrália, publicados no período de 1840-1845.

\section{C2.2 - Australian Museums On Line}

URL: http://www.nma.gov.au/AMIS

Tem por objetivo prover acesso aos recursos culturais existentes nos museus australianos.

Douglas, L. \& Hall, S. Providing access to Australian cultural heritage resources through the Internet. Ariadne, n. 9, 1997.

URL: http://www.ariadne.ac.uk/issue9/ museums/

Descrição dos objetivos e a implantação de uma biblioteca e museu digitais englobando vários organizações australianas.

\section{C2.3 - Australian Parliament House}

URL: http://www.aph.gov.au/

Projeto de biblioteca digital da Parliamentary Library.

\section{C2.4 - Curtin University}

URL: http://www.curtin.edu.au/curtin/ library

Projeto de biblioteca digital da Curtin University.

\section{C2.5 - Indian Ocean Rim Region Virtual Library}

URL: http://www.cowan.edu.au/library/ iorr/home.htm

Projeto cooperativo de várias universidades australianas que tem por objetivo disponibilizar informações digitais relativas aos diversos assuntos dos países banhados pelo Oceano Índico.

Lutley, S. The Indian Ocean Rim Region Virtual Library Project. Ariadne, n. 9, 1997.

URL: http://www.ariadne.ac.uk/issue9/ ocean-rim/
Breve descrição do projeto da biblioteca digital Indian Ocean Rim Region.

\section{C2.6 - Monash University}

URL: http://www.lib.monash.edu.au/ wwwlib/

Projeto de biblioteca digital contendo a coleção de livros reservados biblioteca da Monash University (campus Berwick).

\section{C2.7 - National Library of Australia}

\section{C2.7.1 - Projeto World1}

URL: http://www.nla.gov.au/2/NDIS

Esforço cooperativo entre as bibliotecas nacionais da Austrália e da Nova Zelândia, tem por objetivo a provisão do acesso a documentos digitais de interesse para os dois países.

\section{C2.7.2 -Documentary Images}

URL: http://www2.nla.gov.au/imagecoll/

Projeto de digitalização de aquarelas, pinturas e fotografias.

\section{C2.8-REDD}

URL: http://lib83.library.uq.oz.au/

Projeto de provisão de documento eletrônico, desenvolvido pela University of Queensland, Queensland University of Technology e Griffith University.

Taylor, C. REDD: Regional Electronic Document Delivery Service. Ariadne, n. 9, 1997.

URL: http://www.ariadne.ac.uk/issue9/ redd/

Descrição do projeto REDD para provisão de documento digital a usuários de bibliotecas universitárias australianas.

\section{C2.9 - State Library of Victoria}

URL: http://www.slv.vic.gov.au

Biblioteca digital da coleção de pinturas, desenhos, cartões postais, mapas, livros raros, posters, história oral e outros materiais da coleção da State Library of Victoria. 
Herman, C. \& Kurzeme, I. The future of digitising at the State Library of Victoria, Australia. Ariadne, n. 9, 1997.

URL: http://www.ariadne.ac.uk/issue9/ digitising

Discussão do projeto Multimedia Source Project da State Library of Victoria.

\section{C2.10 - UNILINC}

URL: http://www.unilinc.edu.au

Projeto cooperativo das bibliotecas universitárias australianas que possibilita acesso ao texto completo de artigos de periódicos.

\section{C2.11 - University of Queensland}

\section{C2.11.1 - Hume Image Collection}

URL: http://www.library.uq.edu.au/

Projeto de biblioteca digital de fotografias da família Hume, cobrindo o período de 1870 a 1890.

\section{C2.11.2-Queensland Country Towns Image Project}

URL: http://architect.uq.edu.au/diglib/ index.html

Projeto que visa a digitalização de slides de prédios históricos das cidades de Queensland.

\section{C2.12 - University of Sidney}

URL: http://setis.library.usyd.edu.au

SETIS (Scholarly Electronic Text and Image Service), da University of Sidney, possibilita acesso a documentos digitais nas áreas de Artes e Humanidades.

\section{C3 - Brasil}

\section{3.1 - Programa Prossiga}

URL: http://www.prossiga.cnpq.br/

No Programa Prossiga - Informação e Comunicação para a Pesquisa, vinculado ao Conselho Nacional de Desenvolvimento Científico e Tecnológico (CNPq) existem diversos recursos informacionais e de comunicação pela Internet.
Dentre eles, destaca-se o Repositório de Informações (REI) [URL:http:// www.prossiga.Incc.br/rei.html], que é um diretório de informações de interesse para os pesquisadores brasileiros, com ligações a sítios localizados no país e no exterior.

\section{C4 - Canadá}

\section{C4.1 - McGill University. Music Library of the Future}

URL: http://lecaine.music.mcgill.ca/MFProject/Html/MLF-Home.html

Projeto desenvolvido pela Faculdade de Musica da McGill University, tem por objetivo implementar uma biblioteca digital sobre música, com ênfase na música canadense.

\section{C4.2 - National Library of Canada. Electronic Publication Pilot Project}

URL: http://www.nlc-bnc.ca/e-coll-e/ index-e.html

Projeto, iniciado em 1994, visa a identificar e solucionar os problemas relacionados com o gerenciamento de publicações eletrônicas e coleções em linha.

\section{C4.3 - Université Laval. GEOREP}

URL: http://sirs.scg.ulaval.ca/

Biblioteca digital de dados espaciais contidos em mapas, fotografias aéreas, imagens de sensoriamento remoto e documentos em multimidia.

Proulx, M. J.; Letourneau, F. \& Martel, C. GEOREP: a WWW customizable georeferenced digital library for spatial data. D-Lib Magazine, December 1996. URL: http://www.dlib.org/dlib/december 96/canada/12proulx.html

Descrição do projeto GEOREP para recuperação de informação geográfica.

\section{C5 - Dinamarca}

C5.1 - Jukebox - Music Across Borders

URL: http://www.sb.aau.dk/Jukebox/ edit-report.html
Projeto de desenvolvimento de um arquivo sonoro dos países da Comunidade Européia.

\section{C6 - Espanha}

\section{C6.1 - Archivo General de la Indias (Sevilha)}

Kalstrom, D. Archive project preserves Columbus documents optically. CD-ROM Professional, v. 6, n. 4, p. 135-140, 1993.

Descrição do projeto de digitalização, indexação e recuperação da informação do Arquivo Geral das Índias (Sevilha).

\section{C7 - Estados Unidos}

\section{C7.1 - Carnegie Mellon University}

C7.1.1 - Carnegie Mellon University. Informedia Digital Library

URL: http://www.informedia.cs.cmu.edu/

Projeto integrado de voz e imagem visando à criação de biblioteca digital de vídeo, baseada, principalmente, no acervo da televisão educativa WQED de Pittsburgh.

Christel, M. Addressing the contents of video in a digital library. ACM Workshop on Effective Abstractions in Multimedia, 1995.

URL: http://www.cs.tufts.edu/ isabel/ christel/christel.html

Descreve as dificuldades de incorporar vídeo e áudio nas bibliotecas digitais. Comenta os métodos e técnicas utilizados na busca e a consulta de segmentos de vídeo e áudio. Discute a visualização e a recuperação desses tipos de informação em ambientes de bibliotecas digitais.

Christel, M.; Kanade, T.; Mauldin, M.; Reddy, K.; Sirbu, M.; Stevens, S. \& Watclar, H. Informedia Digital Video Library. Communications of the ACM, v. 28, p. 57-58, April 1995.

Breve descrição do projeto Informedia da Carnegie Mellon University. 
Wactlar, H., Kanade, T., Smith, M., \& Stevens, S. Intelligent access to digital video: The Informedia Project. IEEE Computer, v. 29 , n. 5, p. 46-52, May 1996.

URL: http://www.computer.org/pubs/ computer/dli/r50046/r50046.htm

Relatório, com diversos gráficos, das atividades realizadas pelo projeto Informédia, informando as dificuldades enfrentadas no reconhecimento de voz para o material em aúdio, representação do conteúdo do vídeo para a sua recuperação e a arquitetura da interface do usuário.

\section{C7.1.2 - Carnegie Mellon University. Mercury Project}

Lowry, C. B. \& Richards, B. Courting discovery: managing transition to the virtual library. Library Hi Tech, v. 12, n. 4, p. 7-13, 1994.

Descrição da implantação do projeto Mercury.

Richards, Barbara G. Project Mercury: the virtual library infrastructure at Carnegie Mellon University. In: Saunders, Laverna, ed. The evolving virtual library: visions and case studies. Medford (NJ): Information Today, 1996. p. 67-88.

Mostra a evolução histórica do projeto de biblioteca digital na Carnegie Mellon University (Pittsburgh, PA), incluindo dados sobre a arquitetura e a interface do sistema, as bases de dados geradas localmente e as adquiridas de fornecedores externos, o subsistema de acesso a texto completo de artigos de periódicos.

Troll, D. A. Research on the distributed electronic library. Advances in Library Automation and Networking, v. 5, p. 203277, 1994.

Artigo detalhado sobre o projeto Mercury, analisando, principalmente, os seus aspectos técnicos.

\section{C7.2 - Columbia University}

Oversized Color Images Digitization Project

URL: http://www.columbia.edu/imaging/ html/largemaps/oversized.html
Project Bartleby: the Public Library on the Internet

URL: http://www.columbia.edu/ svls2/

Cartolano, R., Gertz, J., \& Klimley, S. Oversized color images: addressing issues of preservation and access. Sem data.

URL: http://www.columbia.edu/ klimley/ oversized1.html

Relatório final do Oversized Color Image Project. Aborda a digitalização, acesso, controle bibliográfico e avaliação.

Klavans, J. L. New Center at Columbia University for digital library research: fostering interdisciplinary research and bridging cultural clashes. D-Lib Magazine, March 1996.

URL: http://www.dlib.org/dlib/march96/ klavans/03klavans.html

Atividades do Center for Research on Information Access da Columbia University.

\section{C7.3 - Cornell University}

A Cornell University possui diversos projetos em andamento:

Memory of America

URL: http://moa.cit.cornell.edu/

Cornell Digital Library Web Interface

URL: http://OITNET.CIT.CORNELL. EDU:80

Consortium for University Printing and Information Distribution (CUPID)

URL: http://OITNET.CIT.CORNELL. EDU:80/CUPID

Schlabach, M. L. \& Barnes, S. J. The Mann Library gateway system. PublicAccess Computer Systems Review v. 5, n. 1, 1994

URL: http://info.lib.uh.edu/pr/v5/n1/ schlabac.5n1

Descrição do sistema Mann Library Gateway que provê acesso a texto completo de artigos de periódicos e arquivos de dados numéricos.

\section{C7.4 - Emory University. Virtual Library Project}

URL: http://www.emory.edu/VL/vlhome. html
Projeto que visa a ampliar o acesso à informação na Emory University.

\section{C7.5 - IBM}

URL: http://www.software.ibm.com/is/ dig-lib/

Lunin, L.F. IBM announces electronic copyright solutions. Information Today, v, 12, n. 5, p. 1, 3, 5, 1995.

Descrição sucinta da biblioteca digital da IBM e seus projetos na área, enfatizando as técnicas para marcação de texto, encriptação dos dados, sistemas de controle do acesso e de contabilidade, arquitetura do sistema.

\section{C7.6 - Indiana University}

Fenske, D. E. \& Dunn, J. W. The Variations Project at Indiana University's Music Library. D-Lib Magazine, June 1996.

URL: http://www.dlib.org/dlib/june96/ variations/06fenske.html

Descreve o projeto de biblioteca digital de música na Indiana University.

\section{C7.7 - Library of Congress. National Digital Library Project}

A Library of Congress possui os seguintes projetos em andamento:

American Memory

URL: Icweb2.loc.gov/ammen/ammenhome. html

American Special Collection

URL: http://lcweb.loc.gov/spcoll/ spclhome.html

Coountry Studies/Area Handbook Program

URL: http://lcweb.loc.gov/homepage/ country.htmll

Digital Library Collection

URL: http://lcweb.loc.gov/homepage/ digital.html

Electronic Exhibits

URL: http://lcweb.loc.gov/homepage/ exhibits.html

Thomas: Legislative Information on the Internet

URL: http://thomas.loc.gov/ 
Arms, C.R. Historical collections for the National Digital Library: lessons and challenges at the Library of Congress, part 1. D-Lib Magazine, n. 4, April 1996. URL: http://www.dlib.org/dlib/april96/loc/ 04c-arms.html

Apresenta o contexto e a história do National Digital Library Project, enfatizando o processo de digitalização, gerenciamento do projeto, fluxo de trabaIho e descrição dos arquivos.

Arms, C.R. Historical collections for the National Digital Library: lessons and challenges at the Library of Congress, part 2. D-Lib Magazine, n. 5, May 1996. URL: http://www.dlib.org/dlib/may96/loc/ 05c-arms.html

Focaliza os problemas de navegação, interfaces e organização do acervo para uma comunidade diversificada de usuários, o registro bibliográfico, o suporte para pesquisa por assunto, busca em texto completo e as limitações da tela do monitor de vídeo.

Fleischhauer, C., \& Erway. R. L. Observations on the reproduction of various library and archival material formats for access and preservation. Washington (DC), 1992.

URL: http://lcweb.loc.gov/pub/american.memory/white.papers/reprod.txt

Descreve as políticas e os procedimentos de digitalização no projeto da American Memory. Enfatiza os fatores de qualidade de reprodução para os diferentes tipo de mídia e a eficiência da reprodução.

Fleischhauer, C. Organizing digital archival collections: American Memory's experiences with bibliographic records and other finding aids. In: Seminar on Cataloging Digital Documents 1994, Charlottesville, Virginia. Proceedings. 1994.

URL: http://lcweb.loc.gov/catdir/ semdigdocs/carl.html

Descreve as experiências do American Memory Project na organização de uma coleção digital. Enfatiza o registro eletrônico numa biblioteca de grande diversidade de formatos, os problemas de direitos autorais do registro eletrônico, o processo de digitalização e o gerenciamento das atividades.
Lamolinara, G. Metamorphosis of a national treasure. American Libraries, v. 27, p. 31-33, March 1996.

Analisa os esforços realizados pela Library of Congress na construção de um acervo digital.

Library of Congress. National Digital Library Project. American Memory User Evaluation Team. Final report of the American Memory user evaluation 19911993. Washington (DC), 1993. (on-line). URL: http://lcweb2.loc.gov/ammem/ usereval.html

Relatório sobre os dois primeiros anos do projeto (1991-1993) e que teve por objetivos definir a clientela mais apropriada para a biblioteca digital e medir as reações, necessidades e expectativas dos usuários. Conclui que o projeto tem tido boa aceitação por parte de professores e alunos das escolas de segundo grau dos Estados Unidos.

\section{C7.8 - National Aeronautical and Space Agency (Nasa)}

A NASA possui os seguintes projetos em andamento:

Digital Library Tecnology (DLT)

URL: http://dlt.gsfc.nasa.gov/

Information Infrastructure Technology and Applications (IITA)

URL: http://cesdis.gsfc.nasa.gov/hpccm/ iita.hp/iita.html

Public Use of Remote Sensing Data URL: http://rsd.gsfc.nasa.gov/rsd/

Technical Report Server

URL: http://techreports.larc.nasa.gov/ cgi-bin/NTRS

Nelson, M. L. et al. The NASA Technical Report Server. Internet Research: Electronic Network Applications and Policy, v. 5, n. 2, p. 25-36, 1995.

URL: http://tebtre.larc.nasa.gov:80/ltrs/ papers/NASA-95-ir-p25/NASA-95ir-p25.html

Artigo discute a arquitetura e os problemas de implantação do Nasa Technical Report Server (NTRS). O NTRS é uma rede composta de diversas bibliotecas digitais que possibilita acesso a documentos técnicos.
C7.9 - National Library of Medicine (NLM)

Walker, F. \& Thoma, G. Access techniques for document image databases. Library Trends, v. 38, n. 4, p. 751-786, 1990.

Descrição do projeto da NLM de interligar os registros do Medline ao texto completo dos documentos indexados.

\section{C7.10 - Stanford University. Digital Libraries Project}

URL: http://www-diglib.stanford.edu/ diglib/

URL: http://walrus.stanford.edu/diglib/

Projeto, iniciado em 1994, tem objetivo desenvolver tecnologias para implementação de bibliotecas digitais.

Cousins, S.B. A task-oriented interface to a digital library. Working Paper, 1995. URL: http://www-diglib.stanford.edu/diglib/WP/PUBLIC/DOC44.html

O documento é dividido em duas partes. Na primeira parte, está incluído o desenho da interface com o usuário; na segunda, a descrição do protótipo denominado InfoBus.

Reich, V., \& Winograd, T. Working assumptions about the digital library. Working Paper, 1995.

URL: http://www-diglib.stanford.edu/ diglib/WP/PUBLIC/DOC10.html\#what

Descreve os objetivos gerais do projeto, usuários potenciais, conteúdo e equipamentos utilizados.

Stanford Digital Libraries Group. The Stanford Digital Library Project. Communications of the ACM, v. 38, p. 59-60, April 1995.

Breve descrição do projeto de biblioteca digital da Stanford University.

\section{C7.11 - Tufts University}

URL: http://medusa.perseus.tufts.edu/

Biblioteca digital de textos clássicos sobre Grécia e Roma. 
C7.12 - University of California, Berkeley. Digital Library Project

URL: http://sunsite.berkeley.edu

URL: http://elib.cs.berkeley.edu/

Projeto que visa a criação de uma biblioteca digital na área do meio ambiente.

Ogle, V. \& Wilensky, R. Testbed development for the Berkeley Digital Library Project. D-Lib Magazine, July/August 1996.

URL: http://www.dlib.org/dlib/july96/ berkeley/07ogle.html

Descrição dos testes que serão executados pelo projeto.

Van House, N. A., Butler, M. H., Ogle, V., \& Schiff, L. User-Centered iterative design for digital libraries: the Cypress experience. D-Lib Magazine v. 2, n. 2, February 1996.

URL: http://www.dilb.org/dlib/february96/ 02vanhouse.html

Apresenta as experiências, o desenho do projeto, a metodologia e o processo de avaliação utilizados no projeto. Aborda o sistema Cypress de recuperação de imagens. Inclui as principais conclusões do estudo, principalmente aquelas relacionadas com o desenho da interface e as mudanças feitas em decorrência desses resultados.

Van House, N., Butler, M., \& Schiff, L. Needs assessment and evaluation of a digital library environment: the Berkeley experience. Berkeley (CA), University of California, 1996.

URL: http://info.sims.berkeley.edu/ vanhouse/dl96.html

Detalha o desenho do projeto e o seu contexto institucional. Relaciona as principais conclusões feitas a partir do estudo de avaliação, principalmente aquelas ligadas a localização da informação; análise dos dados; disseminação, publicação e reutilização da informação.

\section{C7.13 - University of California, Santa Barbara. Alexandria Project}

URL: http:// alexandria.sdc.ucsb.edu/ Projeto visa à criação de uma biblioteca digital com imagens na área de informação geográfica.
Frew, J., Carver, L., Fischer, C., Goodchild, M., Larsgaard, M., \& Smith, T. The Alexandria rapid prototype: building a digital library for spatial information. 1995 ESRI User Conference. Proceedings.

URL: http://www.esri.com/resources/ userconf/proc95/to300/p255.html

Descreve os testes iniciais realizados para avaliar o projeto, particularmente aqueles ligados a interface com o usuário e a arquitetura da biblioteca digital. Inclui também os planos para o futuro.

Frew, J.; Freeston, M.; Kemp, R. B.; Simpson, J.; Smith, T.; Wells, A. \& Zheng, Q. The Alexandria Digital Library testbed. D-Lib Magazine, July/August 1996. URL: http://www.dlib.org/dlib/july96/ alexandria/07frew.html

Testes realizados no Projeto Alexandria para a recuperação de informação geográfica.

Lasgaard, M. L. \& Carver, L. Accessing spatial data online: Project Alexandria. Information Technology and Libraries, v. 14, p. 93-97, June 1995.

Descreve o projeto Alexandria para a recuperação de informação geográfica.

Smith, T. R. A digital library for geographically referenced materials. Conference on Untangling the Web 1996, Santa Barbara, California. Proceedings. Santa Barbara (CA): University of California, 1996.

URL: http://www.library.ucsb.edu/ untangle/frew.html

Também em: Computers v. 29, p. 5460, May 1996

[URL: http://www.computer.org/pubs/dli/ r50054/r50054.htm]

Descreve os aspectos técnicos e os problemas metodológicos dos principais elementos do projeto enfatizando a sua arquitetura, componentes do catálogo, interface com o usuário e processamento de imagens.

University of California, Santa Barbara. Alexandria Digital Library. Project Team 1996 annual report. Santa Barbara (CA): University of California, 1996.

URL: http://alexandria.sdc.ucsb.edu/ public-documents/annual-report/
Relatório sobre as atividades realizadas pelo projeto até fevereiro de 1996. Inclui também a programação futura. Os principais tópicos abordados são desenvolvimento e testagem de um protótipo para a WWW, estrutura organizacional, recursos humanos, metadados, desenho de interface, avaliação, processamento de imagem, relacionamento com outras entidades e atividades educacionais.

\section{C7.14 - University of California, San Francisco}

URL: http://www.library.ucsf.edu/

Kunze, J. A. ; Warling, B. N. Recent development in Galen II: evolution of a Digital Library for the Health Sciences. D-Lib Magazine, March 1996.

URL: http://www.dlib.org/dlib/march96/ 03galen2.html

Descrição do projeto de biblioteca digital nas áreas de ciências da saúde.

Lucier, R. E. Building a digital library for the Health Sciences: information space complementing information place. Bulletin of the Medical Library Association v. 83, p. 346-350, July 1995.

Analisa a implementação de uma biblioteca digital de ciências da saúde na University of San Francisco.

\section{C7.15 - University of Illinois, Urba- na-Champaign}

Projeto visa à criação de uma biblioteca digital, na área de engenharia, vinculada à Grainger Engineering Library. URL: http://www.grainger.uiuc.edu/dli/

Bishop, A. P. Working towards an understanding of digital library use. D-Lib Magazine v. 1, n. 3, October 1995. URL: http://www.dlib.org/dlib/october95/ 10bishop.html

Aborda os esforços realizados pelos seis participantes do Digital Libraries Initiative (DLI), enfatizando a sincronização das reuniões e a criação de lista dos participantes, visando à otimização do programa comum de pesquisa. Comenta tópicos a serem observados durante a avaliação dos projetos, a saber, desempenho da busca e recuperação da informação, efeitos sobre os usuários e implicações junto às políticas públicas. 
Bishop, A. P., Star, S. L., Neumann, L., Ignacio, E., Sandusky, R. J., \& Schatz, B. Building a university digital library: understanding implications for academic institutions and their constituencies. In: Higher Education and the NII: from vision to reality. Proceedings of the Monterey Conference 1995, Washington, DC, 1995.

URL: http://anshar.grainger.uiuc.edu/dlisoc/monterey.final_copy.html

Relatório de avaliação do projeto abordando o comportamento de busca de informação do usuário, uso do computador, utilização do periódico e da biblioteca por parte dos estudantes de graduação e pós-graduação, professores e estudantes secundaristas. Através de entrevistas e observações, o grupo de pesquisa enfocou o impacto da comunidade digital sobre os indivíduos e instituições.

Schatz, B., Mischo, W., Cole, T., Hardin, J., Bishop, A., \& Chen, H. Federating repositories of scientific literature: the Illinois Digital Library Project. IEEE Computer, v.29, p. 28-36, May 1996.

A. URL: http://www.grainger.uiuc.edu/ dli/ieeecomp.htm

Informa os esforços de pesquisa feitos pelo projeto ressaltando os testes de arquitetura, métodos e técnicas utilizados na indexação dos documentos, avaliação do uso, interface com o usuário e a arquitetura do servidor.

\section{C7.16 - University of Michigan.}

\section{C7.16.1 - University of Michigan. Digital Library Project (UMDL)}

URL: http://www.umdl.umich.edu/

Projeto englobando diversos departamentos da universidade visando pesquisar e desenvolver bibliotecas digitais.

Atkins, D. E.; Birmingham, W. P.; Durfee, E. H.; Glover, E. J.; Mullen, T.; Rundensteiner, E. A.; Soloway, E.; Vidal, J. M.; Wallace, R. \& Wellman, M. P. Toward inquiry-based education throught interacting software agents. Computer, v. 29, p. 69-76, May 1996.

URL: http://www.computer.org/pubs/ computer/dli/r50069/r50069.htm
Analisa os aspectos técnicos relativos ao projeto de construção da biblioteca digital.

Birmingham, W. P., Drabenstott, K. M., Frost, C. O., Warner, A. J., \& Willis, K. The University of Michigan Digital Library: this is not your father's library. Conference on the Theory and Practice of Digital Libraries, 1st., 1994, College Station, Texas. Proceedings. College Station (TX), Texas A \& M University, 1994. URL: http://www.csdl.tamu.edu/DL94/ paper/umdl.html

Descrição do projeto da University of Michigan/Digital Library ligada às áreas de geociências e engenharia espacial. Inclui os princípios da arquitetura do sistema, descrição dos módulos utilizados, protocolos, mecanismos de testes e avaliação do uso.

Birmingham, W. P. An agent-based architecture for digital libraries. D-LIB Magazine, v. 1, n. 7, July 1995.

URLL: http://www.cnri.reston.va.us/ home/dlib/July95/07birmingham.html

Dividido em duas partes, o documento, inicialmente, descreve os serviços propostos pela biblioteca digital; na segunda parte, focaliza as interações entre os diversos módulos do projeto para a provisão dos serviços a seus usuários.

Crum, L. University of Michigan Digital Library Project. Communications of the ACM, v. 38, p. 63-64, April 1995.

Breve descrição do projeto de biblioteca digital da University of Michigan.

Wellman, M., Birmingham, W. P., Durfee, E. H., Rundensteiner, E. A.,

Glover, E., \& Mullen, T. Building the University of Michigan Digital Library. IEEE Computer, special issue on building large-scale digital Libraries, May 1996. URL: http://ai.eecs.umich.edu/people/ wellman/pubs/Building-MDL.html

Descrição técnica do projeto, enfatizando a arquitetura do sistema, formas de busca da informação e utilização dos seus recursos por professores e estudantes.
C7.16.2 - University of Michigan. Humanities Text Initiative (HTI)

URL: http://www.hti.umich.edu/

Warner, B. F. \& Barber, D. Building the digital library: the University of Michigan's UMLIbText Project. Information Technology and Libraries, v. 13, n. 1, p. 20-24, March 1994.

Análise do projeto Humanities Text Initiative da University of Michigan, abordando sua evolução histórica, os recursos informacionais existentes, políticas de acesso, suporte ao usuário e planos futuros.

\section{C7.16.3 - University of Michigan. Internet Public Library (IPL)}

URL: http://www.ipl.org/

Simcox, S. IPL: the Internet Public Library. Ariadne, n. 7, 1997.

URL: http://www.ariadne.ac.uk/issue7/ipl/

Descrição da fase inicial de criação da Internet Public Library (IPL), primeira biblioteca publica digital disponível na Internet, bem como os diversos serviços existentes.

\section{C7.16.4 - University of Michigan. Journal Storage Project (JSTOR)}

URL: http://www.jstor.org/

Projeto de periódicos eletrônicos nas áreas de ciências e artes. Inicialmente estão sendo implementados títulos nas áreas de Economia e História.

\section{C7.17 - University of Pittsburgh. Electronic Text Project}

URL: http://stirner.library.pitt.edu/

Projeto, iniciado em setembro de 1994, visa pesquisar e desenvolver bibliotecas digitais na universidade.

\section{C7.18 - University of Tennessee. Scholar's Workstation Project}

\section{URL: http://scholar.lib.utk.edu/sws/}

Projeto visa a desenvolver biblioteca digital departamental para atender as necessidade de informação dos docentes e estudantes. 


\section{C7.19 - Vanderbilt University}

URL: http://www.mc.vanderbilt.edu/adl/

Biblioteca digital com informações biomédicas.

\section{C7.20 - Virginia Tech}

Fox, E. A.; Eaton, J. L.; McMillan, G.; Kipp, N. A.; Weiss, L.; Arce, E. \& Guyer, S. National Digital Library of Theses and Dissertations. D-Lib Magazine, September 1996.

URL: http://www.dlib.org/dlib/september 96/theses/09fox.html

Comentário sobre a evolução e os problemas enfrentados na implantação de uma biblioteca digital de dissertações e teses.

\section{C7.21 - Xerox}

URL: http://www.parc.xerox.com/

Pesquisas visando ao desenvolvimento de tecnologias aplicadas a bibliotecas digitais.

\section{C7.22 - Yale University. Project Open Book.}

URL:http://www.library.yale.edu/pres/ pobweb.html

Projeto, iniciado em 1991, com a colaboração da Xerox Corporation, com o objetivo de criar uma biblioteca digital contendo 10 mil volumes.

\section{C8 - França}

\section{C8.1 - Bibliothèque Nationale de France}

URL: http://www.bnf.fr

Tem diversos projetos de digitalização de documentos e imagens.

\section{C8.2 - Bibliothèque Publique d'Information (BPI)}

URL: http://www.bpi.fr

Localizada no Centre Georges Pompidou (Paris) a BPI permite acesso a diversos acervos digitais.
C8.3 - Bibliotheque Municipale de Lyon

URL: http://www.bm-lyon.fr

Além do catálogo em linha, provê acesso a livros, periódicos e mapas antigos relativos à história francesa.

\section{C8.4 - Institut de Recherche et Coordination Acoustique-Musique (IRCAM)}

URL : http://www.ircam.fr

Localizado no complexo cultural Georges Pompidou em Paris, contém biblioteca digital sobre música.

\section{C9 - Holanda}

\section{9.1 - Tilburg University}

Geleijnse, H. \& Grootaers, C. Developing the library of the future: the Tilburg experience. Tilburg: Tilburg University Press, 1996. 125 p. ISBN 90-361-9686-8

Obra trata da implantação da biblioteca digital no âmbito da Universidade de Tilburg.

Geleijnse, $\mathrm{H}$. Moving from the digital to the virtual library: the Tilburg experience. IEEE International Forum on Research and Technology Advances in Digital Libraries, ADL'97, May 7-9 1997, Washington (DC). Proceedings. Los Alamitos (CA): IEEE Computer Society, 1997. p. 56-62.

Descrição dos desenvolvimentos realizados na biblioteca digital da Tilburg University no período de 1995-96.

\section{C9.2 - TULIP (Elsevier Science)}

Iniciativa da Elsevier Science Publishers para pesquisar as questões relacionadas com a distribuição eletrônica do periódico científico.

Lynch, C.A. The TULIP project: context, history, and perspective. Library $\mathrm{Hi}$ Tech, v. 13, n. 4, p. 25-27, 1995.

Síntese do projeto TULIP, descrevendo os contextos tecnológico e histórico. O autor analisa o critério de seleção dos títulos de periódicos, obstáculos enfrentados na formatação dos dados, proble- mas organizacionais e os desafios encontrados durante a implementação do projeto dentro das instituições participantes.

Mostert, P. TULIP at Elsevier Science. Library Hi Tech, v. 13, n. 4, p. 27-30, 1995.

Síntese dos principais problemas enfrentados na produção em larga escala de periódicos eletrônicos na Elsevier Science, a saber, formatos, métodos de produção e transmissão dos dados via ftp. O autor conclui que o armazenamento, a velocidade de transmissão e a transferência via Internet são os desafios maiores.

Project TULIP. Tulip final report. URL: http://www.elsevier.nl/info/projects/ trmenu.htm

URL:http://www.elsevier.nt:80/homepage/ about/resproj/tulip.html

Relatório final do projeto TULIP. Inclui a descrição geral do projeto, os problemas técnicos enfrentados pela Elsevier Science e pelas universidades participantes. Analisa os resultados da avaliação pelos usuários, as dificuldades organizacionais e econômicas e o futuro da biblioteca digital. O documento conclui observando que os problemas econômicos, os obstáculos técnicos com equipamentos e as necessidades dos usuários precisam ser levadas em conta na implantação de projeto similar.

Worona, S., Saylor, J. TULIP at Cornell University. Library Hi Tech, v. 13, n. 4, p. 61-4, 1995.

Descrição do projeto TULIP no âmbito da Cornell University. Enfoca os problemas de incorporação do acervo eletrônico do TULIP dentro de uma biblioteca universitária, os métodos de transferência de arquivo e as mudanças para um ambiente digital.

\section{C10 - Japão}

URL: http://www.dl.ulis.ac.jp/

\section{C10.1 - Nara Institute of Science and Technology}

Sunahara, H.; Imai, M.; Yamaguchi, S.; Sato, K.; Oie, Y.; Yokoya, N. \& Chihara, $\mathrm{K}$. Digital video library system of the 
Mandala Library. In: Electronic Library and Visual Information Research Conference, Elvira 3, De Monfort University, Milton Keynes, UK, April 30-May 2, 1996. Proceedings. London: Aslib, 1996. p. 13-16.

Descrição da arquitetura do projeto da biblioteca Mandala, que entrou em fase operacional em abril de 1996.

\section{C11 - Nova Zelândia}

\section{C11.1 - Computer Science Research}

Witten, Ian $\mathrm{H}_{\text {.; }}$ Cunningham, Sally J.; Vallabh, M.; Bell, Timothy C. A New Zealand Digital Library for Computer Science Research. In: Annual Conference on the Theory and Practice of Digital Libraries, 2., June 11-13, 1995, Austin, Texas. Proceedings. College Station (TX): Texas A \& M University, 1995. p. 25-30.

Descrição do projeto de biblioteca digital de relatórios técnicos da área de informática.

\section{C11.2-MELDEX}

McNab, R. J.; Smith, L. A.; Bainbridge, D. \& Witten, I. H. The New Zealand Digital Library MELody inDEX. D-Lib Magazine, May 1997.

URL: http://www.dlib.org/dlib/may97/ meldex/05witten.html

Descrição do sistema MELDEX para recuperação de músicas a partir de notas cantadas num microfone.

\section{C11.3 - New Zealand Digital Library}

URL: timeframes.natlib.gov.nz/

Imagens digitalizadas sobre a história da colonização da Nova Zelândia, do acervo da coleção Alexander Turbul da National Library of New Zealand.

\section{C12 - Reino Unido}

\section{C12.1-ACORN}

URL: http://acorn.lboro.ac.uk/

Gadd, E. Project Acorn: watching those oaks grow... Ariadne, n. 9, 1997.

URL: http://www.ariadne.ac.uk/issue9/ acorn
Breve descrição do projeto ACORN (Access to Course Readings via Networks) de texto completo de artigos de periódicos, esforço cooperativo entre as universidades de Loughborough e Leicester com a empresa Swets.

\section{C12.2 - British Library}

A British Library tem os seguintes projetos em andamento:

CATaloging and Retrieval of Information

Over Networks Applications (CATRIONA)

URL: http://www.bubl.bath.ac.uk/BUBL/ catriona.html

Digitisation of Microfilm

URL: http://portico.bl.uk/access/microfilmdigitisation.html

Electronic Beowulf

URL: http://portico.bl.uk/access/beowulf/ electronic-beowulf.html

Electronic Photo Viewing System

URL: http://portico.bl.uk/access/ photoview.html

Network OPAC

URL: http://portico.bl.uk/access/ network-opac.html

Patent Express Jukebox

URL: http://portico.bl.uk/access/ patents-jukebox.html

Treasures Digitisation

URL: http://portico.bl.uk/access/treasures/ overview.html

Purday, J. The British Library's Initiatives for access projects. Communications of the ACM, v. 38, p. 65-66, April 1996.

URL: http://portico.bl.uk/access/

Breve descrição dos projetos em andamento na British Library.

\section{C12.3 - De Montfort University. ELINOR (Electronic Library Information Online Retrieval)}

Davies, C. Student attitudes and behaviour towards an electronic library. In: Electronic Library and Visual Information Research Conference, Elvira 3, De Monfort University, Milton Keynes, UK, April 30-May 2, 1996. Proceedings. London: Aslib, 1996. p. 101-109.
Avaliação, sob o ponto de vista dos usuários, das dificuldades e facilidades encontradas quando utilizaram a biblioteca digital ELINOR.

Zhao, D. G. \& Ramsden, A. Report on the ELINOR electronic library pilot. Information Services and Uses, v. 15, n. 3, p. 199-212, 1995.

Descrição do projeto ELINOR de biblioteca digital.

\section{C12.4-Edinburgh Engineering Virtual Library (EEVL)}

URL: http://www.eevl.ac.uk/

Biblioteca digital sobre informação em engenharia.

King, I. \& Moffat, M. Evaluation of the EEVL pilot service. September 1996.

URL: http://www.eevl.ac.uk/evaluation/ report.html

Relatório de avaliação do projeto da Edinburgh Engineering Virtual Library.

\section{C12.5 - Electronic Libraries Programme (eLib)}

URL: http://ukoln.bath.ac.uk/elib/ intro.html

Programa governamental, administrado pelo Joint Information Systems Committee, tem por objetivo financiar pesquisas e projetos na área de bibliotecas digitais.

\section{C12.6 - Eurotext}

URL: http://eurotext.ulst.ac.uk/

Carrol, F. Eurotext, a resource bank of learning materials for European studies. Ariadne, n. 9, 1997.

URL: http://www.ariadne.ac.uk/issue9/ eurotext/

Breve descrição do projeto Eurotext, que possibilita acesso a documentos relacionados com a Comunidade Européia.

\section{C12.7 - Scottish Collaborative On-demand Publishing Enterprise (SCOPE)}

URL: http://www.stir.ac.uk/infoserv/scope 
Halliday, L. SCOPE. Ariadne, n. 9, 1997 URL: http://www.ariadne.ac.uk/issue9/ scope/

Aborda as questões de direito autoral e os problemas logísticos derivados da publicação sob demanda nas bibliotecas universitárias.

\section{C12.8 - University of Bristol}

Williams, J.; Crisp, J.; Lock, A. \& Burnett, C. Development of image libraries for network distribution. In: Electronic Library and Visual Information Research Conference, Elvira 3, De Monfort University, Milton Keynes, UK, 1996. Proceedings. London: Aslib, 1996. p. 17-25.

Análise dos problemas enfrentados na montagem do Bristol Biomedical Image Project. Os problemas comentados são os relativos à captação e acesso às imagens na área biomédica.

\section{C13 - Suécia}

\section{C13.1 - European Legislative Virtual Library (ELVIL)}

URL: http://www.sub.se/sam/elvil.htm

Projeto em desenvolvimento na Universidade de Estocolmo, tem por objetivo a criação de uma biblioteca digital sobre política e legislação européia.

\section{C14 - Vaticano}

Mintzer, F. C., Boyle, L. E., Cazes, A. N., Christian, B. S., Cox, S. C., \& Giordano, F. P. Towards online, world wide access to Vatican Library Materials. IBM Journal of Research and Development, v. 40, n. 2, p. 139-162, 1996.

Descrição do projeto da biblioteca digital do Vaticano, incluindo informações sobre características do sistema, fluxo de trabalho, escaneamento, equipamentos utilizados e recuperação da informação.

\section{D - Construção da biblioteca digital}

Nesta parte, estão incluídas referências sobre os principais aspectos técnicos, tanto da ciência da informação como da Informática, ligados à implementação de uma biblioteca digital.
D1 - Arquitetura do sistema

Arms, W. Y. Key concepts in the architecture of the digital library. D-Lib, July 1995.

URL: www.dlib.org

URL: http://www.cnri.reston.va.us/home/ dlib/July95/07arms.html

Aborda os principais conceitos ligados à arquitetura de bibliotecas digitais.

Arms, W. Y.; Blanchi, C. \& Overly, E. A. An architecture for information in digital libraries. D-Lib Magazine, February 1997.

URL: http://www.dlib.org/dlib/february97/ cnri/02arms1.html

Longo artigo, de cunho técnico da área de informática, sobre arquitetura de bibliotecas digitais.

Kling, R., \& Elliott, M. Digital library design for usability. Annual Conference on the Theory and Practise of Digital Libraries, 1., 1994, College Station, Texas. Proceedings, 1994. p. 146-155

Apresenta cinco modelos de sistemas computacionais que poderão ser úteis na construção da biblioteca digital.

Nurnberg, Peter J.; Furuta, Richard; Legget, John J.; Marshall, Catherine C.; Shipman, Frank M. Digital libraries: issues and architecture. In: Annual Conference on the Theory and Practice of Digital Libraries, 2., 1995, Austin, Texas. Proceedings. College Station (TX): Texas A \& M University, 1995. p. 147-153

O documento apresenta uma taxonomia dos elementos da biblioteca digital e suas implicações na arquitetura do sistema.

\section{D2 - Conversão de dados e Escaneamento [Scanning]}

Saffady, W. Digital library concepts and technologies for the management of library collections: an analysis of methods and costs. Library Technology Reports, v. 31, n. 3, p. 223-383, 1995.

Na parte inicial do artigo, o autor apresenta definições de biblioteca digital e a história dos principais projetos em andamento. A parte principal do documento enfoca a implementação de pro- jetos de digitalização (OCR), abordando hardware, software, conversão de documentos e cálculo dos custos envolvidos.

\section{D3 - Marcação de textos [incluindo SGML e HTML]}

Cole, T. ; Kazmer, M. SGML as a component of the digital library. Library High Tech, v. 13, n. 4, p. 75-90, 1995

Dividido em duas partes, a primeira descreve as características do SGML e sua importância para o ambiente em rede, comentando os problemas que deverão ser considerados pelos bibliotecários quando da implantação do seu uso. A segunda parte enfoca os problemas vinculados a busca, recuperação e visualização.

Corthouts, J. \& Philips, R. SGML: a librarian's perception. Electronic library, v. 14, n. 2, p. 101-110, April 1996.

Análise de diversos projetos de bibliotecas digitais que utilizam a SGML para o controle bibliográfico, acesso à informação e apresentação de texto completo.

Cover, Robin. SGML web page.

URL: http://www.sil.org/sgml/sgml.html

Página que contem documentos, bibliografia e ligações relacionadas com a Standard Generalized Markup Language (SGML).

Hockey, Susan. Developing access to electronic texts in the Humanities. In: Saunders, Laverna, ed. Evolving virtual library: visions and case studies. Medford (NJ): Information Today, 1996. p. 119-133.

Comenta as experiências de digitalização de textos nas áreas de ciências humanas e sociais, enfatizando a necessidade de padrões para a digitalização e indexação dos documentos digitais.

Price-Wilkins, J. Just-in-time conversion, just-in-case collections: effectively leveraging rich document formats for the WWW. D-Lib Magazine, May 1997.

URL: http://www.dlib.org/dlib/may97/ michigan/05pricewilkin.html 
Descrição do serviço de marcação de textos da University of Michigan Digital Library.

Sperberg-McQueen, C. M.; Burnard, Lou, ed. Guidelines for electronic text encoding and interchange. Chicago: University of Illinois at Chicago, 1994. $2 \mathrm{v}$. URL: http://www.iuc.edu/orgs/tei [página do Text Encoding Initiative]

Documento importante, contendo as normas para marcação de textos.

\section{D4 - Desenvolvimento de coleções}

Ackerman, M. S.; Fielding, R. T. Collection development in the digital library. Annual Conference on the Theory and Practice of Digital Libraries, Austin (Texas), 1995. Proceedings. College Station (TX): Texas A \& M University, Department of Computer Science, 1995. p. 39-47.

URL: http://csdl.tamu.edu/DL95/papers/ ackerman/ackerman.html

Os autores mostram que a manutenção da coleção dentro de uma biblioteca digital será uma de suas tarefas críticas. Apresentam diversos mecanismos (MOMspider e WebLookout) que poderão executar o gerenciamento das coleções.

\section{D5 - Catalogação}

Caplan, P. Cataloging Internet resources. The Public-Access Computer Systems Review, v. 4, n. 2, p. 61-66, 1993.

URL: http://www.nlc-bnc.ca/documents/ libraries/cataloging/caplan.txt

Descrição das atividades que possibilitaram a criação do campo 856 no registro MARC. Autor enfatiza a necessidade de inclusão da localização do documento eletrônico no formato MARC.

Corrado, E. Annotated Bibliography on Cataloging Internet Resources. 1996.

URL: http://www.scils.rutgers.edu/ ecorrado/cataloging/index2.html

Bibliografia anotada sobre a catalogação de fontes da Internet.

Hoogcarspel, Annelise. Guidelines for cataloging monographic electronic texts a the Center for Electronic Texts in the Humanities. New Brunswick (NJ):
Princeton University, Center for Electronic Texts in the Humanities, 1994. 38 p. (CETH Technical Report, n. 1)

URL: http://cethmac.princeton.edu/ Docs/catguid.ps

Documento importante, contendo as normas para a catalogação de documentos eletrônicos.

Levy, D.M. Cataloging in the digital order. In: Conference on the Theory and Practice of Digital Libraries, 2., Austin, Texas, June 1995. Proceedings. College Station (TX): Texas A \& M University, 1995. p. 31-37.

URL: http://csdl.tamu.edu/DL95/papers/ levy/levy.html

Examina a natureza e o papel da catalogação no ordenamento das estruturas informacionais na era digital.

Olson, N.B. Cataloging Internet Resources: A manual and practical guide. 1995.

URL: http://www.oclc.org.oclc/man/ 9256cat/toc.htm

Instruções para aqueles que utilizam o manual da OCLC intitulado "Bibliographic formats and standards". Inclui descrição bibliográfica e acesso.

Shieh, J. Does it really matter? The cataloging format, the sequential order of note fields, and the specifics of field 856. OCLC Internet Cataloging Project Colloquium. Sem data.

URL: http://www.oclc.org/oclc/man/ coloq/shieh.htm

Comenta as normas de catalogação das fontes da Internet utilizadas na biblioteca da University of Virginia. Analisa especialmente o formato para seriados, a ordem dos campos $5 x x$ nos diferentes registros catalográficos (OCLC e VIRGO) e a utilização do campo 856 do registro MARC.

\section{D6 - Classificação/Indexação}

Desire WP3

URL: http://www.ukoln.ac.uk/metadata/ DESIRE/classification/

Relatório que discute o papel dos esquemas de classificação na descrição dos recursos da Internet.
Mitchell, S. Library of Congress Subject Headings as subject terminology in a virtual library: The INFOMINE example. In: Untangling the Web 1996, Santa Barbara, California. Proceedings.

URL: http://www.library.ucsb.edu/ untangle/smitch.html

Descreve as razões para a utilização no projeto Infomine da lista de cabeçalhos de assuntos da Library of Congress (LCSH), suas técnicas, hardware e software utilizados no projeto.

\section{D7 - Metadados}

BIBLINK

URL: http://www.ukoln.ac.uk/BIBLINK/ wp $1 / \mathrm{d} 1.1$

Relatório sobre o fluxo de informação, de formatos de metadados, entre os editores e as agências nacionais de controle bibliográfico.

Dempsey, Lorcan.; Weibel, Stuart L. The Warwick Metadata Workshop: a framework for the deployment of resource description. D-Lib, July 1996.

URL: http://www.dlib.org/dlib/july96/ 07contents.html

Comentário sobre as decisões da reunião de Warwick sobre metadados.

IFLA Metadata Resources.

URL: http://www.nlc-bnc.ca/ifla/II/ metadata.htm

Excelente fonte de recursos informacionais sobre metadados.

Lagoze, Carl. The Warwick framework: a container architecture for diverse sets of metadata. D-Lib, July 1996.

URL: http://www.dlib.org/dlib/july96/ 07contents.html

Discussão dos aspectos de arquitetura utilizados em metadados.

Metadata Workshop II, April 1-3 1996. URL: www.oclc.org:5046/oclc/research/ conferences/metadata2/metadata.html

Informações sobre o evento sobre metadados realizado na University of Warwick (UK), em 1-3 de abril de 1996. 
Weibel, Stuart L. Dublin core.

URL: http://purl.org/metadata/dublin core

Homepage sobre metadados que provê importantes ligações a diversas organizações.

\section{D8 - Referência}

Kelly, J. S. \& Robbins, K. Changing roles for reference libraries. Journal of Library Administration, v. 22, n. 2/3, p. 111-121, 1996.

Aborda os fatores tecnológicos e seus impactos potenciais nos serviços de referência.

Myers, J. E. Reference services in the virtual library. American Libraries, v. 25, n. 7, p. 634-638, July/August 1994.

Simulando um diálogo eletrônico, a autora imagina diversos serviços de referência que poderão ser executados na futura biblioteca digital.

\section{D9 - Recuperação da informação [Z39.50, interface, segurança de acesso]}

Anderson, L. C.; Lotspiech, J. B. Rights management and security in the electronic library. Bulletin of the American Society for Information Science, v. 22, n. 1, p. 21-23, 1995.

Descrição dos sistemas de gerenciamento de acesso e segurança utilizados na ISI Electronic Library Project. Detalha a arquitetura do sistema, gerenciamento dos direitos dos usuários, autenticação e criptografia do acesso, autenticação dos documentos e endereço dos usuários.

Dillon, A. What is the shape of information? Human factors in the development and use of digital libraries. Allerton Institute, 37., 1995, Monticello, Illinois. Proceedings. 1995.

URL: http://edfu.lis.uiuc.edu/allerton/95/ s4/dillon.html

Focaliza as conceitualizações de navegação dos usuários no espaço informacional eletrônico.
Marchionini, G. User-centered methods for library interface design. Allerton Institute, 37., 1995, Monticello, Illinois. Proceedings, 1995.

URL: http://edfu.lis.uiuc.edu/allerton/95/ s4/marchio.html

Mostra o processo de desenho da interface da biblioteca digital incluindo as característica básicas que deverão ser observadas. Enfatiza a importância de se levar em conta as necessidades dos usuários e não as limitações do hardware e/ou do software.

Poulter, Alan. Building a browsable virtual library. Aslib Proceedings, v. 46, n. 4, p. 151-155, June 1994.

Mostra a possibilidade de se fazer consulta a "estantes digitais" através de terminal de computador.

Rao, R.; Pedersen, J. O.; Hearst, M. A.; Mackinlay, J. D.; Card, S. K.; Masinter, L. Rich interaction in the digital library. Communications of the ACM, v. 38, n.4, p. 29-40, 1995

Os autores mostram que a aplicação de interfaces na biblioteca digital irá facilitar, de forma eficiente, a utilização de distintos métodos de busca e visualização da informação.

\section{D10 - Direitos autorais}

Arnold, K. The body in the virtual library: Rethinking scholarly communication. Journal of Electronic Publishing, v. 1, n. 1, 1995.

URL: http://www.press.umich.edu/jep/ works/arnold.body.html

O documento discute os futuros modelos, dentro de um ambiente digital, da comunicação científica e a proteção dos direitos intelectuais. Nesses cenários a comunicação científica e os papéis a serem desempenhados pela editora universitária e da biblioteca serão diferentes dos atuais.

Barlow, J.P. The economy of ideas: A framework for rethinking patents and copyrights in the digital age. Wired, v. 2, n. 3, March 1994.

URL: http://www.nlc-bnc.ca/documents/ infopol/copyright/jpbarlow.htm
Artigo apresenta idéias sobre o a propriedade intelectual na era digital.

Copyright and Intellectual Property Resources of the IFLA,

URL: http://www.nlc-bnc.ca/ifla/II/ cpyright.htm

Página da IFLA sobre direitos autorais. Inclui texto integral de muitos documentos.

Library of Congress. Copyright Office; Electronic Registration, Recordation \& Deposit System (CORDS).

URL:http://lcweb/copyright

Página do Escritório de Copyright da Library of Congress.

Lyons, P.; Garrett, J. . Toward an electronic copyright management information system. Journal of the American Society for Information Science, v. 44, n. 8, p. 68-73, 1993.

Exame do gerenciamento dos direitos autorais de obras literárias num ambiente eletrônico e as maneiras pelas quais este processo pode ser automatizado.

Mullin, D.I. The First Amendment and the Web: The Internet porn panic and restricting indecency in cyberspace. Untangaling the Web 1996. Proceedings. Santa Barbara, California, 1996.

URL: http://www.library.ucsb.edu/untangle/mullin.html

Discute a existência de material considerado indecente ou pornográfico na Internet e a legislação norte-americana, de 1996, sobre o assunto (Communications Decency Act).

National Research Council. Rights and responsibilities of participants in networked communities. 1994.

URL: http://www.nap.edu/nap/online/rights/

Discussão das questões éticas e legais relacionadas com a comunicação eletrônica. Aborda a livre expressão, vandalismo eletrônico, propriedade intelectual e privacidade. Conclui que as futuras normas sociais no ambiente digital serão baseadas na legislação atual e nos novos padrões sociais.

Okerson, A. Who owns digital works? Scientific American, v. 275, n. 1, p. 80 84, July 1996.

URL: http://www.sciam.com/0796issue/ 0796okerperson.html 
Discute os problemas de direito autoral, no âmbito dos Estados Unidos, relacionados com as publicações eletrônicas

Perritt, H. Knowbots, permission headers and contract law. Conference on Technological Strategies for Protecting Intellectual Properties in the Networked Multimedia Environment 1993.

URL: http://www.nlc-bnc.ca/documents/ infopol/copyright/perh2.txt

Documento detalhado sobre a proteção da propriedade intelectual na biblioteca digital. Apresenta sugestões para a proteção dos direitos autorais, principalmente com a implementação do permission headers que indica o usuário potencial, inclusive com assinatura e contrato eletrônicos.

Rosenbaum, H. (1996). In the trenches of the digital revolution: intellectual freedom and the 'public' digital library. American Society for Information Science, MidYear Meeting, 18-22 May 1996, San Diego (California). Proceedings. Medford: Information Today, 1996. p. 163-176. URL: http://silver.ucs.indiana.edu/ hrosenba/Papers/ASIS963.html

Discussão sobre os problemas enfrentados pelas bibliotecas em relação ao acesso a Internet, abordando a liberdade intelectual, livre expressão, privacidade, acesso e propriedade intelectual.

Samuelson, P. Copyright and digital libraries. Communications of the ACM, v. 38, n. 4, p. 15-21, 1995.

Apresenta a evolução histórica do direito autoral e mostra que, no ambiente das bibliotecas digitais, o modelo "pague pelo uso" (pay-per-use) poderá ser uma das melhores alternativas para a utilização de material que possui copyright.

Solan, Olivier de. Les documents informatiques et l'avenir du depot legal. Bulletin des Bibliothèques de France, $v$. 40, n. 4, p. 28-32, 1995.

Análise da legislação francesa de direito autoral em relação ao documento eletrônico.

\section{D11 - Preservação da informação digital}

The Commission on Preservation and Access and the Research Libraries

Group. (1995). Task force on archiving of digital information proposed charge. 1995. URL: http://www.oclc.org:5046/ $\sim$ weibel/archtf.html

URL: http://www.rlg.org/ArchTF/ [texto final do documento apresentado em maio de 1996]

Documento importante, pois contém os critérios para a preservação do documento digital.

Graham, P.S. Intellectual preservation and electronic intellectual property. IP Workshop Proceedings 1994.

URL: http://www.cni.org/docs/ima.ipworkshop/www/Graham.html

http://aultnis.rutgers.edu/texts/ cpaintpres.html

Dividido em duas partes o documento discute: 1) meios de preservação, enfatizando o problema da obsolescência tecnológica; 2) preservação do conteúdo intelectual.

Lynch, Clifford. The integrity of digital information: mechanics and definitional issue. Journal of the American Society for Information Science, v. 45, n. 10, p. 737-744, 1994.

Excelente artigo sobre preservação da informação digital abordando a autenticidade, integridade e preservação do conteúdo intelectual.

Preserving Access to Digital Information (PADI)

URL: http://www.nla.gov.au/dnc/tt2001/ padi/padi.html

Excelente repositório de informação sobre preservação em seus mais variados aspectos.

Rothenberg, Jeff. Ensuring the longevity of digital documents. Scientific American, p. 24-29, January 1995.

Autor mostra a necessidade de ser preservada a informação digital mesmo com as constantes mudanças tecnológicas.

\section{E - Fontes de informação}

\section{E1 - Monografias}

Fox, Edward A. Source book on digital libraries; technical report. Virginia Tech, December 1993. $410 \mathrm{p}$.

URL: gopher://fox.cs.vt.edu/11/DL URL: http://fox.cs.vt.edu/DLSB.html

Obra importante para entendimento das atividades realizados nos Estados Unidos na área de biblioteca digital no período de 1990-1993. Dividida em oito capítulos, a obra inclui o documento para a construção da National Electronic Library (cap. 1); comentários sobre os encontros profissionais sobre o assunto realizados em 1992 e 1993 (cap. 2-4) e dos projetos em andamento (cap. 5-6). Além disso, nos capítulos 7-8, incluiu bibliografia e lista das abreviaturas.

Kessler, J. Internet digital libraries: the international dimension. Boston: Artech House, 1996. 265 p. ISBN 0-89006-875-5

Com uma abordagem internacional, a obra analisa algumas questões pouco discutidas na literatura. São elas as barreiras lingüísticas, políticas e o processo de normalização em diferentes países.

Pitkin, Gary M. The national electronic library: a guide to the future for library managers. Westpot (CT): Greenwood Press, 1996. 192 p. (ISBN 0-313-296138) Preço: US\$55.00

Contendo 12 contribuições, a obra está dividida em duas partes: a primeira, sobre a National Electronic Library, enfoca os projetos norte-americanos de bibliotecas digitais; a segunda parte trata dos serviços e planejamento das atividades bibliotecárias dentro do novo cenário eletrônico.

Saunders, Laverna M., ed. The evolving virtual library: visions and cases studies. Medford (NJ): Information Today, 1996. 153 p. (ISBN 1-57387-013-7) Preço: US\$39.50

Inclui oito trabalhos relacionados com redes automatizadas e bibliotecas digitais. 


\section{E2 - Principais periódicos}

Ariadne, the Web version. Dundee (Scotland): University of Abertay, University Library, v. 1 -, 1996-. ISSN 1361-3200

URL: http://www.ariadne.ac.uk/

Periódico eletrônico, inclui artigos e notícias sobre a Internet, biblioteca digital e publicação eletrônica.

Computers in libraries. Medford: Information Today, v. 1-, 1988-,10 números por ano. ISSN 1041-7915 URL: http://www.infotoday.com/

Titulo que enfatiza a automação de bibliotecas, com boas avaliações sobre hardware e software.

Current cites. Berkeley: University of California, Library Technology Watch Program, v. 1 -, 1990 - . ISSN 10602356

URL: http:/sunsite.berkeley.edu/ CurrentCites/

Periódico eletrônico, inclui bibliografia anotada de artigos de periódicos, livros e documentos eletrônicos sobre publicação eletrônica, multimidia, hipermidia, redes e bibliotecas digitais.

D-lib Magazine. Reston (VA): Corporation for National Research Institute (CNRI), v. 1 -, July 1995- . ISSN 1082-9873 URL: www.dlib.org

URL: www.cnri.reston.va.us/home/ dlib.html

Periódico eletrônico, inclui artigos, notícias e calendário de eventos relacionados com todos os aspectos da biblioteca digital. Título de alta relevância.

Diginews. v. 1 -, April 1997-

URL: www.rlg.org/preserv/diginews

Periódico eletrônico preparado pelo Research Library Group, em cooperação com a Cornell University Library, contém novidades sobre preservação da informação digital.

Electronic Library: the international journal for minicomputer, microcomputer and software applications in libraries. Medford (NJ): Learned Information, v. 1-, Jan. 1983.
Com ênfase em aplicativos e equipamentos utilizados na automação de bibliotecas, contém alguns artigos sobre biblioteca digital.

Electronic Library Research. London: ASLIB, v. 1 -, 1997 (?)

A ASLIB anunciou o próximo lançamento desse periódico.

IBM Journal of Research and D evelopment. Armonk (NY): IBM, v. 1-, Jan. 1957 - .

URL:http://www.almaden.ibm.com/journal

Periódico de informática que também inclui artigos sobre biblioteca digital.

IBM Systems Journal. Armonk (NY); IBM, v. 1 -, Sept. 1962-

URL:http://www.almaden.ibm.com/journal

Periódico de informática que também inclui artigos sobre biblioteca digital, especialmente aqueles ligados a projetos desenvolvidos pela IBM ou que utilizam recursos dessa empresa.

INFOBITS. Research Triangle Park (NC): Institute for Academic Technology, v. 1-, July 1993-. ISSN 1071-5223

URL: http://www.iat.unc.edu/infobits/ infobits.html/

Periódico eletrônico que inclui resumos selecionados sobre tecnologia de informação.

Initiatives in Digital Information. Ann Arbor (MI): University of Michigan, Digital Library Program, v. 1 -, June 1995 - .

URL:http://www.lib.umich.edu/libhome/ IDINews/

Periódico eletrônico da University of Michigan que inclui artigos sobre a informação digital nos seus mais diversos aspectos.

International Journal on Digital Libraries. Berlin: Springer Verlag, v. 1-, 1997. Trimestral, ISSN $1432-5012$ (impressa), ISSN 1432-1300 (em linha). Assinatura: US $\$ 260.00$

URL: htpp://link.springer.de [acesso gratuito para quem assina a versão impressa]
Primeiro periódico, sob a forma impressa, específico sobre biblioteca digital, tem por objetivo "servir de veículo de disseminação de artigos de pesquisa de alta qualidade que tratem das questões relacionadas com a produção da informação digital, gerenciamento e uso, redes de alta velocidade e conectividade, intemporabilidade e integração de sistemas, pessoas, perfis, necessidades, segurança e privacidade dos indivíduos e das transações comerciais e o processo efetivo do comércio na Idade da Informação" (introdução, v. 1, p. 2).

Internet Reference Services Quartely. Haworth Press, v. 1 -, 1996 - .

URL: http://www.cableskill.edu/service/ Irc/irsq

Periódico impresso que inclui artigos e notícias sobre tecnologias e recursos informacionais da Internet.

InterNIC News. Internet Network Information Center

URL: http://rs.internic.net/nic-support/ nicnews

Boletim eletrônico de notícias sobre a Internet.

Journal of Electronic Publishing. Ann Arbor; University of Michigan.

URL: http:'//www.press.umich.edu/jep/

Periódico eletrônico, inclui artigos sobre biblioteca digitais, a ênfase, porém é sobre publicação eletrônica.

Journal of Internet Cataloging. Haworth Press, v. 1 -, 1996 - .

URL: http://www.libraries.psu.edu/ iasweb/personal/rob/jic/jic.html

Periódico impresso que inclui artigos sobre classificação, acesso e organização da informação digital.

Library Hi Tech. Ann Arbor (MI): Pierian Press, v. 1-, 1983-, 10 numeros por ano.

Excelente fonte para informações correntes sobre automação de bibliotecas, contendo também artigos sobre bibliotecas digitais.

National Digital Library Program. Washington (DC): Library of Congress, v. 1 -, October 1995-, mensal. ISSN 1083-3978 URL: http://lcweb.loc.gov/ndl/per.html 
Noticiário sobre as atividades do programa de biblioteca digital da Library of Congress.

Public Access Computer System News. Houston (TX): University of Texas, University Library, v. 1 -, March 1990 -. URL: http://info.lib.uh.edu/pacnews.html

Periódico eletrônico, inclui notícias sobre sistemas computacionais em bibliotecas.

Public Access Computer System Reviews. Houston (TX); University of Texas, University Library, v. 1 -, 1990 - .

URL: http://info.lib.uh.edu/pacrev.html

Periódico eletrônico, inclui artigos sobre sistemas computacionais em bibliotecas. Nota: também disponível sob a forma impressa (Chicago: American Library Association, v. 1-, 1992 -, quadrimestral).

The Scout Report. Herndon (VA): Internet Network Information Center (INTERNIC), v. 1 - .

URL: http://rs.internic.net/scout/report/

Periódico eletrônico, boletim semanal sobre as novidades na Internet.

\section{E3 - Reuniões técnicas específicas}

Os eventos abaixo seguem a ordem cronológica, do mais antigo para o mais recente.

\section{E3.1 - Eventos de 1994}

ELVIRA-1 Electronic Library and Visual Information Research, 1., May 1994, UK. Proceedings. London: Aslib, 1995. 173 p. ISBN 0-85142-347-7

Anais contendo os 15 trabalhos apresentados no evento.

Annual Conference on the Theory and Practice of Digital Libraries, I., June 19-21, 1994, College Station, Texas (US). Proceedings. College Station (TX): Texas A \& M University, Department of Computer Science, 1994. 221 p.

URL: http://abgen.tamu.edu/DL94

URL: http://bush.CS.tamu.edu/dl94/

README.html

URL: http://www.csdl.tamu.edu/DL94/
Anais contendo os 43 trabalhos que foram apresentados.

Adam, Nabil; Bhargava, B.; Yesha, Y., ed. Digital libraries, current issues. New York: Springer Verlag, 1995. $321 \mathrm{p}$. (ISBN 3-54-59282-2) Preço: US\$56.00 URL:http://superbook.bellcore.com/ DBRG/DL94/

Anais dos 17 trabalhos apresentados no Digital Libraries Workshop DL'94, Newark, New Jersey (US), May 19-20 1994.

\section{E3.2 - Eventos de 1995}

Digital Libraries Conference, Singapore, Singapore Information Technology Institute, March 27-28, 1995.

URL:http://www.iti.gov.sg/iti-info/event/ conference/Dlib.htm/

Evento organizado pela Library Association of Singapore.

ELVIRA-2 Electronic Library and Visual Information Research, 2., May 1995, UK. Proceedings. London: Aslib, 1995. 152p. ISBN 0-85142-354-X

Anais contendo os 15 trabalhos apresentados no evento.

Follet Lectures Series.

URL: http://ukoln.bath.ac.uk/follett/ intro.html

Série de palestras sobre bibliotecas digitais organizada pelo UK Office of Library and Information Networking (UKOLN).

Annual Conference on the Theory and Practice of Digital Libraries, 2., June 1113, 1995, Austin, Texas (US). Proceedings. College Station (TX): Texas A \& M University, Department of Computer Science, 1995. 205 p. (preço: US\$25.00) URL: http://csdl.tamu.edu/DL95

URL: http://bush.cs.tamu.edu/dl95/ README.html

Anais contendo os 28 trabalhos que foram apresentados no evento. Inclui, no final, índices de autores e assuntos.

Allerton Institute

URL: http://edfu.lis.uiuc.edu/allerton/95
Anais dos trabalhos apresentados durante o $37^{\text {th }}$ Allerton Institute que teve como tema central o desenho e avaliação de bibliotecas digitais.

International Symposium on Digital Libraries 1995, Tsukuba Science City, Japan, University of Library and Information Science, August 22-25, 1995.

URL: http://www.DL.ulis.ac.jp/ISDL95/ proceedings/

Anais dos trabalhos apresentados no simpósio realizado em 1995 em Tsukuka (Japão).

Symposium on Reconnecting Science and Humanities in Digital Libraries, 19-21 October 1995, University of Kentucky.

URL: http://www.uky.edu/ kiernan/DL/ symp.html

Evento que discutiu os problemas computacionais e metodológicos das bibliotecas digitais nas áreas das humanidades.

\section{E3.3 - Eventos de 1996}

Information, National Policies and International Infrastructure, January 28-30, 1996.

URL: http://kswww.harvard.edu/ itbspp/ gii2pap.html

Anais do simpósio realizado na J. F. Kennedy School of Government da Harvard University que discutiu políticas nacionais de informação e redes internacionais.

Social Aspects of Digital Libraries. Los Angeles (CA): University of California/ Los Angeles, Graduation School of Education \& Information Studies, 1996. URL: http://www.gslis.ucla.edu/DL/

Anais do seminário sobre os aspectos sociais da biblioteca digital realizado em fevereiro de 1996 na University of California/Los Angeles.

ACM International Conference on Digital Libraries, $1^{\text {st }}$., March 20-23, 1996, Bethesda, Maryland (US). Proceedings. New York: ACM, 1996 URL: http://fox.cs.vt.edu/foxinfo.html

Anais da conferência patrocinada pela Association for Computing Machinery (ACM), em março de 1996. 
ELVIRA-3 Electronic Library and Visual Information Research, 3., 30 April-2 May 1996, UK. Proceedings. London: Aslib, 1996. 183 p. ISBN 0-85142-383-3

Anais contendo os 18 trabalhos apresentados no evento.

Seminar on Electronic Publishing URL: http://www.vtt.fi/inf/nordep/ proceedings/epsem96/ohjelma.html

Anais do seminário promovido pelo Nordic Centre of Excellence for Electronic Publishing, realizado em Lund (Suécia), em 11 de junho de 1996.

SIGIR'96: Workshop on Networked Information Retrieval, August 22, 1996. URL: http://ciir.cs.umass.edu/nir96

Anais contendo os oito trabalhos apresentados sobre recuperação da informação e bibliotecas digitais.

Allerton-96

URL: http://edfu.lis.uicc.edu/allerton96

Evento, organizado pela School of Library and Information Science da University of Illinois (Urbana), em 27-29 de outubro de 1996, que teve como tema central a pesquisa sobre bibliotecas digitais.

\section{E3.4 - Eventos de 1997}

IEEE ADL'97: International Conference on Advances in Digital Libraries, May 7-9 1997, Washington, DC (US). Proceedings. Los Alamitos (CA): IEEE Computer Society Press, 1997. 153 p. ISBN 0-8186-8010-5

URL: http://cesdis.gsfc.nasa.gov/adm/ adl97/adcall.html (informações sobre a programação do evento)

Anais contendo os 14 trabalhos apresentados no evento.

ELVIRA-4 Electronic Library and Visual Information Research, 4., 6-8 May 1997, Milton Keynes (UK).

URL: http://www.iielr.dmu.ac.uk/ELVIRA/ ELVIRA4/ (programa e outras informações; os anais serão publicados pela ASLIB).

Colloquium on Digitizing Photographic Collections, June 7-9 1997.

URL: http://www.rit.edu/ andpph/ brochure.pdf
Beyond the beginning: the global digital library Conference, June 16-17, 1997, London, UK).

URL: http://www.ukoln.ac.uk/events/global-digital-library/

ACM International Conference on Digital Libraries, 2., July 23-26 1997, Philadelphia, PA(US),

URL: http://www.sis.pitt.edu/ diglib97/

Congreso Internacional sobre información electronica y bibliotecas digitales, 23-26 de setiembre de 1997, San Jose, Costa Rica.

URL: http://www.una.ac.cr/bibl

European Conference on Research and Advanced Technology for Digital Libraries, 1-3 September 1997, Pisa (Itália).

URL: http://www.area.pi.cnr.it/ErcimDL/

International Symposium on Research, Development \& Practice in Digital Libraries, November 18-21 1997, University of Library and Information Science, Tsukuba (Japan).

URL: http://www.DL.ulis.ac.jp/ISDL97/

\section{E3.5 - Eventos de 1998}

IEEE International Conference on Advances in Digital Libraries, 2., Santa Barbara (California), 22-24 April 1998.

URL: http://www.alexandria.ucsb.edu/ conferences/ADL98

\section{E4 - Lista de discussão}

Biblioteca virtual (bib-virtual).

E-mail:bib-virtual@buriti.ibict.br

Lista de discussão sobre bibliotecas digitais criada no Brasil, pelo IBICT, em 1997.

Digital Librarians Discussion List (DigLibns) URL: http://sunsite.berkeley.edu/DigLibns/

Lista de discussão, criada em dezembro de 1996, com a finalidade de discutir questões práticas e os procedimentos para a construção de bibliotecas digitais.

DIGLIB. IFLA Digital Libraries Research E-mail: listserv@infoserv.nlc-bnc.ca
Lista de discussão da IFLA sobre bibliotecas digitais, é gerenciada pela $\mathrm{Bi}$ blioteca Nacional do Canadá.

hyperjournal-forum

E-mail:mailbase@mailbase.ac.uk

Lista de discussão sobre periódico eletrônico.

JISC Electronic Libraries Research Programme (lis-elib).

E-mail:mailbase@mailbase.ac.uk

Lista de discussão sobre os projetos de bibliotecas digitais no Reino Unido.

\section{E5 - Grupos/centros de estudos}

De Montford University. International Institute of Electronic Library Research (IIELR)

URL: http://ford.mk.dmu.ac.uk/ Documents/instit.html/

Organização que tem por objetivo a promoção do conhecimento e a implementação de bibliotecas eletrônicas. Esse instituto tem promovido as conferências anuais ELVIRA (cf. lista de eventos).

IEEE Digital Library Task Force.

URL: http://cimic.rutgers.edu/iee-dltf. html

Grupo de especialistas, criado em 1995 pela IEEE Computer Society, para discutir os aspectos técnicos da área de informática relacionados à biblioteca virtual.

Texas A \& m University. Center for the Study of Digital Libraries (CSDL) URL; http://www.csdl.tamu.edu/

Criado em 1995, pela Texas A \& M University, tem por objetivo pesquisar os aspectos teóricos e práticos da biblioteca digital. O Centro já organizou duas conferências profissionais.

\section{E6 - Cursos/treinamento}

Digital Imaging for Libraries and Archives. Cornell University Library, Department of Preservation and Conservation, September 14-19 1997. E-mail:preserve@cornell.edu 
Curso, com duração de uma semana, sobre uso de tecnologia de imagem digital em bibliotecas e arquivos.

Digital Tool Kit. University of Michigan, School of Information.

URL: http://www.si.umich.edu/hp/ institutes/

Cursos rápidos (2-5 dias) oferecidos no período de maio-agosto de 1997.

International Summer School on the Digital Library, Tilburg University (Holanda). URL: http://cwis.kub.nl/ ticer/

Cursos sobre biblioteca digital, com duração de duas semanas, realizados em agosto de 1996 e 1997.

Worshop on Digital Libraries, University Library and Information Science (ULIS), Tsukuba (Japão).

URL: http://www.dl.ulis.ac.jp/DLW-E/EDLworkshop.html

Desde 1994, a ULIS tem sido promovidos cursos rápidos sobre bibliotecas digitais.

\section{Digital libraries: an annotated international bibliography}

\begin{abstract}
International annotated bibliography about digital libraries. The following aspects are covered: a) visionaries, authors that wrote about the library of the future during the period of 1945-1985; b) concepts of digital library; c) major on-going projects in Germany, Australia, Brasil, Canada, Denmark, Spain, United States, France, Netherlands, Japan, New Zealand, United Kingdom, Sweden and Vatican; c) tecnical aspects related to the construction of the digital library, such as: system architecture, data conversion and scanning, text enconding, collection development, cataloging, classificacation and indexing, metadata, reference, information retrieval, copyright and the preservation of digital information; e) major information sources: monographs, periodical titles, technical meetings, discussion lists, groups and center of studies, courses and trainning.
\end{abstract}

\section{Keywords}

Digital library; Digital library architecture; Data conversion; Scanning; Text marking; Electronic text encoding; SGML; Collection development; Cataloging; Classification, Indexing; Metadata, Reference, Information Retrieval; Z39.50; Copyright; Digital information; Preservation of information.

\section{CONCLUSÃO}

O assunto biblioteca digital, conforme se pode depreender pela bibliografia apresentada, tem tido um crescimento acentuado desde 1994. De três congressos profissionais, especificamente dedicados ao tema em 1994, o número cresceu para cinco em 1995 e para oito, em 1997. Dois novos títulos de periódicos (sendo um eletrônico, D-Lib Magazine, e outro impresso, International Journal on Digital Libraries) passaram a divulgar artigos sobre pesquisas e projetos em andamento. Além disso, listas de discussão, artigos de periódicos e monografias demonstram que o tema está em pleno crescimento. Esse crescimento, inicialmente restrito aos Estados Unidos e Reino Unido, começa a atingir outros países.

Biblioteca digital, também denominada pelos britânicos de biblioteca eletrônica, é uma área que tem grande futuro na ciência da informação. Espera-se que parte do seu conteúdo seja incorporado aos currículos das escolas brasileiras de arquivologia, biblioteconomia e ciência da computação. Assim, esta bibliografia pretendeu colaborar na divulgação do assunto e motivar estudos e pesquisas na área.
Murilo Bastos da Cunha

Universidade de Brasília

Departamento de Ciência da Informação e Documentação

70910-900 Brasília, DF

murilobc@guarany.unb.br 